\author{
Piero Amenta \\ (avvocato del Tribunale apostolico della Rota Romana)
}

\title{
Diritto processuale penale canonico e inveramento del principio fondamentale del diritto alla difesa ${ }^{1}$
}

\author{
Canonical criminal procedural law related to the principle of self-defense: \\ a comparative analysis
}

\begin{abstract}
The article compiles an analysis of canonical criminal procedural law tracing it in the first place to the lack, in the canonical system, of a constitutional law, which Paul VI had deemed necessary to be adopted when, in the aftermath of Vatican Council II, he decided to promote that Lex Ecclesiae Fundamentalis, later abandoned under the pontificate of John Paul II. The work makes use of a comparative analysis with Italian criminal procedural law and compares the procedural institutes of one and the other legal system to help understand the breadth and consistency of the gaps and shortcomings of canonical procedural law.
\end{abstract}

SOMMARIO: 1. Introduzione: dichiarazione d'intenti, obiettivo e metodo - 2. L'assenza di una costituzione nella Chiesa e problematica connessa - 3. L'ordinamento canonico e i suoi limiti nella tutela dei diritti individuali - 4. Un esempio paradigmatico: il processo per l'imposizione di un decreto penale.

\section{1 - Introduzione: dichiarazione d'intenti, obiettivo e metodo}

Ogni ordinamento giuridico e persino ogni singolo sistema processuale presenta imperfezioni e insufficienze. L'ordinamento giuridico di uno stato o di una organizzazione internazionale è sempre perfettibile, come tutte le cose umane, e soggiace alla legge dell'evoluzione: un ordinamento giuridico si evolve perché si evolve il complesso dei valori fondamentali a cui fa riferimento la società che lo adotta. Talvolta l'evoluzione si attua a seguito di precise scelte di politica giudiziaria, talaltra perché il complesso normativo, col trascorrere del tempo, risulta confliggente con i valori di riferimento dei consociati. Tali imperfezioni e insufficienze finiscono per riverberarsi nella pratica forense, che è quell'attività di cui il consociato ha più immediata percezione, soprattutto in ambito penale, quello che più di

\footnotetext{
${ }^{1}$ Contributo sottoposto a valutazione. Article peer evaluated.
} 
tutti gli altri tocca da vicino la sua vita personale, familiare e sociale. Compito dello studioso, come anche dell'operatore del diritto che non si fermi allo svolgimento di una mera attività burocratica, è rilevare le insufficienze e le carenze, perché se ne possano individuare gli adeguati correttivi, e offrire nel contempo al Legislatore le ragioni per eventuali interventi di riforma.

Il diritto canonico, pur non essendo un diritto statale in senso stretto, non fa eccezione da questo punto di vista.

Scopo di questo studio è quello di dare un fondamento scientifico, dal punto di vista delle scienze giuridiche, alle tante perplessità che pone il sistema penale e processuale canonico, intraviste e percepite in lunghi anni di attività accademica e professionale ma finora rimaste essenzialmente a livello di intuizione. Negli ultimi decenni, le perplessità e persino le carenze e le insufficienze sono emerse ancor più decisamente, accentuatesi a causa di una più intensa pratica penale dovuta alle note $\mathrm{e}$ meno note vicende giudiziarie inaugurate dopo la promulgazione del motu proprio Sacramentorum sanctitatis tutela, del 2010, a opera di papa Benedetto XVI, spesso, anche se non esclusivamente, legato alle vicende di veri o presunti abusi a danno di minori. L'intensificazione negli ultimi tempi di una pratica penale tutto sommato piuttosto sporadica nel passato, ha dunque fatto vieppiù emergere interrogativi, perplessità e insufficienze diffuse. L'errore, a giudizio di chi scrive, sarebbe però liquidare tali insufficienze come il prodotto di problematiche di carattere contingente, come l'impreparazione degli operatori del diritto o la scarsità dei mezzi istruttori a disposizione degli organi giurisdizionali della Chiesa. Pur non disconoscendo la presenza e il peso di tali contingenze, sono del parere che le carenze che si rilevano nel diritto penale e processuale canonici sono radicate in un sistema che non appare ancora pienamente adeguato a garantire il rispetto dei diritti proclamati in linea di principio dalla Chiesa cattolica. Anzi, mi spingo ad affermare, con il proposito di motivare nel prosieguo del presente scritto, che l'ordinamento canonico, che pure ha il merito storico e comunemente riconosciuto di aver apportato agli ordinamenti penali statuali un contributo di indubbio valore morale e di umanizzazione dei sistemi penali del passato, oggi patisce un grave ritardo di civiltà e non di rado sconfessa nella pratica forense ciò che proclama solennemente in linea di principio ${ }^{2}$.

\footnotetext{
2 Faccio osservare - ad esempio - che la recentissima riforma del codice penale canonico voluta da papa Francesco e che sta per entrare in vigore riporta lodevolmente la menzione, al canone introduttivo, del principio della presunzione di non colpevolezza,
} 
I canonisti generalmente trattano diversamente il problema, giustificando gli scostamenti notevoli che il diritto canonico presenta rispetto ai codici statuali con una specificità ordinamentale, dovuta all'origine e ai fini che persegue il diritto della Chiesa e che non sono assimilabili a quelli degli stati di diritto moderni; il fatto, cioè, che esso scaturisca da una società sui generis, quella ecclesiale, che non può essere assimilata alle società civili, conferirebbe al diritto canonico caratteristiche peculiari e ciò sarebbe sufficiente a giustificare l'assenza di taluni istituti giuridici o la peculiarità degli altri istituti che compongono il sistema. Ma se questo è vero e giusto per le norme che riguardano l'amministrazione dei sacramenti, il modo di organizzare gli ordini e istituti religiosi, il modo di organizzare le circoscrizioni ecclesiastiche sul territorio e via dicendo, insomma, se questo è vero per materie che sono tipicamente ecclesiali, non lo è e non dovrebbe esserlo per una materia, quella penale, che tocca da vicino le vicende umane e che può compromettere, talvolta in modo irreversibile, la vita personale, familiare e sociale degli individui coinvolti. Infatti, le insufficienze e le criticità di un sistema, di qualsiasi sistema penale, finiscono per riverberarsi nella pratica forense, che è quell'attività di cui il cittadino e il fedele hanno più immediata percezione. Dunque, la peculiarità e la specificità del diritto canonico non sembra sufficiente a giustificare l'assenza di istituti che presiedono alla tutela concreta dei

che mancava nella legislazione appena abrogata, nonostante essa non sia antichissima, risalendo al 1983. A questo proposito, si impongono due osservazioni: il fatto stesso che solo oggi, a ben 75 anni di distanza dalla Convenzione europea dei diritti dell'uomo (art. 6) e ad oltre 250 anni dalla sua teorizzazione a opera di Cesare Beccaria, il diritto canonico penale abbia introdotto espressamente tale principio fondamentale, ormai patrimonio comune di quasi tutte le società umane sparse sul globo, è di per sé già un fatto significativo. Dunque, non è fuori luogo parlare di ritardi di civiltà in rapporto al diritto canonico; in secondo luogo, la pur lodevole ma tardiva menzione non sembra affatto essersi inverata negli istituti penali e processuali canonici e rischia di restare anche questa una mera affermazione di principio. In un recentissimo convegno sulla riforma del diritto penale canonico, S. Ecc. Arrieta, mentre si dichiarava in disaccordo con coloro che hanno visto nella riforma di papa Francesco una sorta di irrigidimento della normativa penale, poi ammetteva che le nuove disposizioni hanno puntato a una contrazione degli spazi di discrezionalità che la normativa del 1983 prevedeva e ha cercato di recuperare le altre finalità della legge penale canonica, finora eccessivamente concentrata sulla sola finalità dell'emendazione del reo. Il prosieguo di questo nostro contributo dimostrerà, spero in modo convincente, la discutibilità di quell'analisi e di quelle affermazioni dell'illustro oratore, visto che la finalità dell'emendazione del reo nel diritto penale canonico è e resta un'affermazione di principio che non trova spazi concreti di tutela a livello processuale (Giornata di studio su Diritto e giustizia penale, del 26 novembre 2021, organizzata dalla cattedra di Diritto ecclesiastico dell'Università telematica «G. Fortunato» di Benevento, i cui atti sono in via di pubblicazione). 
principi fondamentali di civiltà, massimamente di quello della presunzione di non colpevolezza e della possibilità effettiva di difendere nel foro competente la propria onorabilità e il proprio buon nome, dunque il sacrosanto diritto all'autodifesa.

Per raggiungere il risultato che ci siamo prefissi, adotteremo il metodo comparativistico. E dal momento che è ovviamente impossibile operare una comparazione con tutti gli ordinamenti civili dell'epoca moderna, ci limiteremo a una comparazione tra gli istituti giuridici canonici e il sistema giuridico italiano, inteso quale complesso di principi costituzionali e di istituti giuridici ancorati ai dettami della nostra carta costituzionale. Non ci sfuggono le problematiche e le carenze legate a una scarsa armonizzazione del sistema giuridico penale e processuale italiano: se, infatti, il codice di rito, promulgato nel 1988, ha potuto tener presenti i principi costituzionali e ne costituisce la traduzione operativa in ambito giurisdizionale, non così dicasi per il codice penale, promulgato in epoca fascista (1930) e dunque anteriore alla Costituzione vigente, del 1947. Non sono poche le norme della nostra carta costituzionale che non trovano adeguata corrispondenza nel codice penale, nato in seno a una cultura di stampo autoritario e poco permeata dai grandi principi che oggi sono comune acquisizione delle società democratiche. Tale consapevolezza ci porterà - nell'operare la comparazione di cui sopra - a fare un più costante riferimento al codice di rito, invece che al codice sostantivo, in quanto più aderente ai principi e alle norme costituzionali.

\section{2 - L'assenza di una costituzione nella Chiesa e problematica connessa}

Un primo problema di partenza nella nostra riflessione riguarda l'assenza di una costituzione materiale nella Chiesa cattolica. E pur vero che l'assenza di una costituzione scritta non vuol dire assenza di principi supremi riconosciuti come costituzionali e ispiratori dell'ordinamento giuridico $^{3}$. Lo stesso, infatti, accade per alcuni stati moderni nei quali l'assenza di un testo costituzionale scritto non impedisce un assetto democratico della nazione e un sistema di equilibrio dei poteri. Per questo, gli studiosi di diritto costituzionale canonico distinguono tra costituzione

3 Un insigne costituzionalista italiano annota che "Una costituzione, in senso sostanziale, si rinviene necessariamente in qualsiasi ordinamento; e si suol parlare, infatti, correttamente, in dottrina, di una costituzione della Chiesa cattolica e di una costituzione dell'ordinamento internazionale [...]" (V. CRISAFULLI, Lezioni di diritto costituzionale, I, $2^{a}$ ed., Cedam, Padova, 1970, p. 86. 
materiale e costituzione sostanziale ${ }^{4}$ e assegnano il carattere di costituzione sostanziale, o reale, ai principi che la Chiesa riconosce come supremi del proprio ordinamento. Bisogna purtuttavia riconoscere che l'assenza di una costituzione scritta rende più difficile l'individuazione dei principi fondamentali a cui deve ispirarsi la vita della comunità. Infatti, i principi fondamentali che la Chiesa riconosce come costituzionali scaturiscono da una pluralità di fonti di diverso genere e peso: la sacra Scrittura, la dottrina degli Apostoli, i dettami dei Padri della Chiesa e dei concili generali, lo Costituzioni Apostoliche, e via dicendo. Una sintesi dei principi ordinamentali provenienti da una tale molteplicità di fonti è certamente molto ardua. La difficoltà di enucleare i principi supremi di riferimento per l'ordinamento giuridico è reale, se Paolo VI, all'indomani del Concilio Vaticano II e in procinto di intraprendere la riforma del Codice di diritto canonico, si pose il problema e, dopo aver sottoposto la questione alla valutazione di una Commissione, decise che fosse opportuna la redazione di un testo scritto che individuasse precisamente $\mathrm{i}$ principi costitutivi della comunità ecclesiale. Opera tanto più necessaria, per il fatto che la Chiesa presenta un'eccezionale diversità di riti, di lingue, di culture, di spiritualità, di tradizioni giuridiche e di strutture molto superiore alle diversità che possono coesistere sul territorio di uno stato unitario e che postulano naturalmente la necessità di un nucleo centrale intorno al quale organizzare la vita ecclesiale ai vari livelli e nelle varie aree geografiche. Nasce così l'idea della redazione di un testo, poi denominato Lex Ecclesiae Fundamentalis: un progetto che, dopo un iter lungo e complesso, fu definitivamente abbandonato. Si scelse invece che alcuni articoli di essa confluissero nel nuovo Codice di diritto canonico, promulgato poi da papa Giovanni Paolo II nel 1983. Vivacissimo fu il dibattito che diede luogo la vicenda della Lex, sia in sede dottrinale che sui mezzi di comunicazione di massa. Non è luogo a occuparci di esso e facciamo volentieri rimando a opere specifiche e dettagliate ${ }^{5}$. Una delle

4 M. DEL POZZO, Introduzione alla scienza del diritto costituzionale canonico, Edusc, Roma, 2015, pp. 181-205. Non si tratta di una distinzione originaria e propria del diritto canonico, perché mutuata o parallela a quella delle dottrine costituzionalistiche. Si veda, ad esempio, V. CRISAFULLI, Lezioni, cit., p. 86.

${ }_{5}^{5}$ Per un iter dettagliato circa i primi passi del testo a partire dal 1965 fino agli anni settanta, si può utilmente consultare L. ROSA, La Lex Ecclesiae Fundamentalis. Il lungo e faticoso iter di un progetto, in Problemi e prospettive di diritto canonico, a cura di E. CAPPELLINI, Queriniana, Brescia, 1977, pp. 51-70; lo stesso articolo pubblicato in Agg. Soc., fasc. 5/28, maggio 1977, pp. 319-337. Per una cronistoria completa del progetto e le problematiche canonistiche a esso connesse, si veda: G.P. MONTINI, Il diritto canonico dalla A alla Z. L. Lex Ecclesiae fundamentalis, in Quaderni di diritto ecclesiale, 14, 2001, pp. 89- 
prime questioni sollevate fu quella della legittimità e dell'opportunità di un tale testo, la sua natura e il suo scopo, nonché il suo rapporto con i due Codici canonici, quello della Chiesa Latina e quello delle Chiese orientali cattoliche. Tuttavia, non sembra affatto, dalla lettura degli atti del tempo, che il progetto sia stato abbandonato per obiezioni circa l'opportunità di dare anche alla Chiesa una carta fondamentale con valore essenzialmente costituzionale, al pari delle costituzioni moderne. Le obiezioni che giunsero al papa da tutti i vescovi del mondo ai quali il testo fu inviato in lettura, riguardarono non la legittimità della presenza di un testo costituzionale né l'opportunità della sua adozione nella Chiesa: le riserve si concentrarono piuttosto sulla qualità del testo provvisorio approntato dalla Commissione e, dunque, sui suoi contenuti. Ragion per cui ci sembra di poter concludere che non è in discussione l'opportunità della presenza nella Chiesa una legge fondamentale con rango costituzionale, quanto piuttosto il problema è definirne i contenutí. Dunque, un'eventuale obiezione all'esistenza di un testo di rango costituzionale nella Chiesa, motivata dalla specificità dell'ordinamento canonico, sarebbe difficilmente sostenibile.

Altro problema è appunto quello che invece riguarda i suoi contenuti. La definizione dei contenuti dipende dalla risposta alla domanda se un testo fondamentale nella Chiesa cattolica debba (o avrebbe dovuto) avere i caratteri delle costituzioni moderne e perseguire gli stessi fini, tra cui quello precipuo della tutela dei diritti fondamentali del fedele cristiano. Lascio agli studiosi di diritto costituzionale canonico la risposta alla domanda.

Per quanto riguarda il tema che mi sono proposto, mi pare di poter affermare che, nonostante le riconosciute specificità dell'ordinamento della Chiesa, non ripugna a un'eventuale costituzione ecclesiale

112.

6 Il Cardinale Felici, che era presidente della Commissione di riforma del Codice, espressamente provocato sulla questione, confidò ai Padri componenti la Commissione di riforma del Codice che - morto inopinatamente papa Luciani, che non ebbe il tempo di esaminare la questione - il nuovo pontefice (Giovanni Paolo II) l'aveva incaricato di costituire una Commissione ad hoc che si occupasse solamente di chiarire il problema dell'opportunità di promulgare la Lex Ecclesiae Fundamentalis e di delinearne i contenuti. Il Cardinale così descrisse le conclusioni della Commissione: "Haec Commisssio coadunata est, discussit de hac re et sub aspectu oecumenico, et maioritas duae tertiae partis - fuit pro opportunitate Legem Fundamentalem promulgandi" (Atti della sessione plenaria IV del 23 ottobre 1981, in PONTIFICIUM CONSILIUM DE LEGUM TeXTIBUS INTERPRETANDIS, Congregatio Plenaria diebus 20-29 octobris 1981 habita, Typis Poliglottis Vaticanis, 1991, pp. 365-366). 
l'eventuale introduzione di norme che presiedano a una legittimazione del potere e ne definiscano i limiti, cioè l'introduzione di una regolamentazione dell'esercizio del potere pubblico, che poi è lo scopo principe delle costituzioni degli stati moderni. Infatti, le costituzioni si sono diffuse in tutti gli stati moderni nel momento del loro passaggio dalla forma di stato assoluto allo stato liberale e democratico: in esse dovevano essere enunciati i nuovi principi dell'ordinamento statale e il modo di esercizio del potere pubblico. Dunque, la mancanza di una costituzione scritta che regolamenti l'esercizio del potere pubblico nella Chiesa, soprattutto in relazione ai diritti fondamentali della persona, costituisce una seria difficoltà per l'ordinamento di approntare un sistema che inveri $\mathrm{i}$ principi riconosciuti come supremi: principi che, in tutte le costituzioni moderne, costituiscono un argine alla tendenza naturale di ogni potere di debordare ed eccedere i limiti che le proprie funzioni comportano, finendo inevitabilmente per invadere spazi che non gli sono dovuti e che appartengono alla sfera privata.

Limitazione e regolamentazione dell'esercizio del potere pubblico, insieme ai mezzi della tutela dei diritti fondamentali della persona umana contro ogni invadenza e ogni possibile violazione, è lo scopo fondamentale della presenza delle costituzioni negli stati moderni ${ }^{7}$. Così concepita, una costituzione scritta non contraddice alla specificità e alla natura della Chiesa e del diritto che ne scaturisce, anzi, sarebbe ancora più necessaria proprio per il fatto che la Chiesa cattolica non riconosce la separazione dei poteri come teorizzata dal Montesquieu ma piuttosto si ispira al principio contrario, quello della concentrazione dei tre poteri legislativo, giudiziario e amministrativo, quale espressione dell'unica potestas sacra che deriva dal sacramento e dalla missione canonica. Orbene, per la natura propria della potestà nella Chiesa, come descritta dai cann. 333 e $375^{8}$ del Codex iuris canonici, che prevede appunto una

7 L'affermazione si riferisce principalmente all'elaborazione teoretica sul costituzionalismo moderno operata dal Kelsen. Una sintesi del suo pensiero è possibile leggere in V. CRISAFULLI, Lezioni, cit., p. 94 ss.

8 "Romanus Pontifex, vi sui muneris, non modo in universam Ecclesiam potestate gaudet, sed et super omnes Ecclesias particulares earumque coetus ordinariae potestatis obtinet principatum, quo quidem insimul roboratur atque vindicatur potestas propria, ordinaria et immediata, quae in Ecclesias particulares suae curae commissas Episcopi pollent" (can. 333, § 1). E ancora: "Episcopi ipsa consecratione episcopali recipiunt cum munere sanctificandi munera quoque docendi et regendi [...]" (can. 375, § 2). Dunque, in forza della consacrazione, i vescovi ricevono la potestà di santificare, insegnare e governare, potestà che esercitano principalmente nella diocesi loro assegnata; il Romano Pontefice, insieme ai tria munera comuni agli altri vescovi, riceve, al momento 
concentrazione dei poteri, appare quanto mai necessario creare un sistema per arginare i possibili abusi e restringere quegli spazi di discrezionalità che possono tramutarsi in arbitrio. E sempre possibile infatti, in una qualsivoglia comunità - e la comunità ecclesiale non fa eccezione - la presenza di espressioni autoritarie, di illegittimità, di arbìtri, che non trovano - come spesso accade nel sistema canonico - adeguati correttivi. Ciò accade in misura molto maggiore che nelle società che godono di un testo in cui sono precisamente delineati i limiti dell'esercizio del potere pubblico, a salvaguardia dei diritti individuali e della sfera privata.

Pur non conoscendo, dunque, l'ordinamento della Chiesa cattolica la separazione dei poteri propria degli stati di diritto, l'eventuale presenza di un testo costituzionale scritto non comprometterebbe - ad avviso di chi scrive - quell'irrinunciabile unità della potestas sacra che è elemento specifico e anch'esso costituzionale della comunità cattolica. Infatti, lo stesso ordinamento canonico prevede lo sdoppiamento tra munus ed esercizio dello stesso (can. 127) e in concreto prevede una serie di organismi diversi, a livello diocesano come a livello centrale, di natura giurisdizionale o amministrativa, ciascuno dotato di competenze proprie e deputati all'esercizio della potestà di governo ai vari livelli: le Congregazioni romane, i tribunali della Sede Apostolica, che esercitano in concreto una potestà che appartiene in maniera propria e ordinaria al Romano Pontefice; le curie diocesane, i tribunali interdiocesani, il sistema vicariale (vicari generali ed episcopali), che esercitano la potestà di governo propria del vescovo nella chiesa particolare a lui affidata. Se dunque, per il principio dell'unità della potestas sacra non è possibile accogliere nell'ordinamento ecclesiale il principio della separazione dei poteri, tuttavia dal punto di vista eminentemente pratico, sia per ragioni organizzative e sia per ragioni di opportunità, non vedo impedimento all'adozione di una pluralità di funzioni distribuite tra organismi diversi ed esercitate da soggetti diversi, se tale pluralità di funzioni sia ben disciplinata dalla legge e rispondente ai principi fondamentali della

dell'accettazione dell'elezione, anche il munus primatiale, o petrino, che esercita con potestà propria, ordinaria e immediata non solo nella propria diocesi ma in tutte le diocesi della terra. Le recenti polemiche sulla validità o meno della rinuncia al pontificato di papa Benedetto XVI mostrano chiaramente l'insufficienza delle prescrizioni codiciali, tanto che da più parti si auspica un approfondimento giuridico-canonico della questione. Mi è giunta notizia che recentissimamente è stato costituito un gruppo di studio che dovrebbe favorire gli scambi tra canonisti e la riflessione comune sul problema dello status giuridico del vescovo di Roma, in vista di una sua possibile, futura migliore definizione a livello normativo (www.progettocanonicosederomana.com). 
costituzione della Chiesa. Ciò permetterebbe un esercizio ordinato del potere pubblico da parte dell'autorità costituita, senza le commistioni e le invasioni di campo a cui si assiste oggi, in assenza di quei meccanismi di razionalizzazione del potere, comuni ai più avanzati modelli di stato e di governo.

\section{3 - L'ordinamento canonico e i suoi limiti nella tutela dei diritti individuali}

Chiedo la venia del lettore per una premessa così lunga - ma a mio avviso del tutto indispensabile - sul problema costituzionale. Non avremmo potuto affrontare correttamente il discorso sui limiti del processo penale canonico e la sua inadeguatezza a garantire il diritto alla difesa se non partendo da quei limiti negativi imposti al potere pubblico nelle costituzioni moderne che si risolvono in garanzie di spazi di libertà per il cittadino e definiscono i contorni della correttezza dei rapporti "politici" che intercorrono tra governanti e governati. Se il rapporto tra ordinamento giuridico e costituzione può apparire un problema del tutto estraneo all'intento che ci siamo proposti esponendo le presenti riflessioni, al contrario - in realtà - i due argomenti sono strettamente connessi. Le lacune e le carenze del sistema canonico, soprattutto quello penale, sono strettamente legate alla mancanza di una legge costituzionale perché sono, lo ripetiamo, carenze strutturali e non legate solamente ad aspetti contingenti. A mo' di esempio, citiamo il problema della possibilità di un sindacato di costituzionalità delle leggi, che manca del tutto nell'ordinamento canonico ${ }^{9}$ proprio perché non è possibile, com'è noto,

\footnotetext{
${ }_{9}^{9}$ Attualmente, il Pontificio Consiglio dei testi legislativi esercita, come prescritto dalla Costituzione Apostolica vigente sulla Curia Romana, Pastor bonus, all'art. 155, il compito dell'interpretazione autentica delle norme legali universali. Inoltre ha il compito della recognitio dei decreti generali delle conferenze dei vescovi e della conformità alle leggi universali dei decreti generali e delle leggi particolari emanati da autorità inferiori al Romano Pontefice (artt. 157-158). Dunque, com'è ovvio, il Pontificio Consiglio non esercita un vero potere di sindacato di costituzionalità che comporti l'abrogazione della vigenza di leggi contrarie alle leggi universali o ai principi supremi dell'ordinamento. Il testo ufficiale della Costituzione Apostolica Pastor Bonus, del 28 giugno 1988 si trova in Acta Apostolicae Sedis, vol. LXXX (1988), pp. 841-912. È significativo quanto affermato da J. Herranz, un tempo Presidente del Pontificio Consiglio: "è questa la prima volta nella storia della Chiesa che viene affidata stabilmente a un organismo della Santa Sede una competenza simile a quella che negli ordinamenti statali hanno le Corti Costituzionali" (il corsivo è nel testo originale di J. HERRANZ, Il Pontificio Consiglio della Interpretazione dei testi Legislativi, in La Curia Romana nella Costituzione Apostolica Pastor Bonus, a cura di P.A.
} 
ipotizzare un tale meccanismo se non in presenza di un testo costituzionale di riferimento. Tuttavia, nell'ordinamento canonico, come in ogni ordinamento giuridico, è sempre possibile che una legge o disposizione sia in contrasto con i principi di rango costituzionale; è sempre possibile che una legge emanata dal Romano Pontefice o da autorità a lui inferiori possano risultare confliggenti con principi supremi dell'ordinamento. Oggi, di fatto, qualora un sinodo provinciale o diocesano, oppure una conferenza di vescovi o qualsiasi altro organo dotato di potere legislativo emani norme derogatorie o addirittura contrarie alla legge universale in assenza di un'espressa previsione di legge che lo permetta, non è possibile attivare alcun sindacato che non sia il solo ricorso al Romano Pontefice. È altresì non raro che accada, e di fatto accade, che alcuni organismi amministrativi di rango apicale, come le Congregazioni romane, deroghino a leggi universali con il semplice strumento di una lettera circolare ${ }^{10}$.

Entrando ora finalmente nell'ambito di interesse centrale di questo scritto, cioè l'ambito processual-penalistico, teniamo anzitutto presente una prima specificità dell'ordinamento canonico, che non prevede una distinzione netta tra codice sostantivo e codice di rito. Le norme processuali sono contenute nel settimo libro del Codice, il quale non distingue le norme rituali penali da quelle relative ai processi contenziosi: il "processo contenzioso ordinario" è il modello unico e paradigmatico per ogni tipo di processo, salva l'integrazione di esso con alcune norme speciali per alcuni tipi di processo, tra cui anche quello penale. Questa peculiarità si risolve in un'assenza concreta di norme speciali di rito che presiedono alla celebrazione di un processo penale: infatti, le norme

BONNET e C. GULLO, LEV, Città del Vaticano, 1990, p. 478). La competenza è detta simile perché il Pontificio Consiglio esercita alcuni compiti che possono essere in qualche modo assomigliare a quello degli organismi statuali che esercitano il giudizio di conformità delle leggi ai principi supremi, ma non si tratta di un compito che può essere accostato a quello delle corti costituzionali, perché non esiste e non può esistere un vero sindacato di costituzionalità in assenza di un testo costituzionale di riferimento.

${ }^{10}$ Così in G.P. MONTINI, Il diritto canonico dalla A alla Z, cit., p. 92. Citiamo anche il caso dell'Istruzione Dignitas connubii, del 25 gennaio 2005, sulla cui forza obbligante si è tanto dibattuto e poi, di fatto, accettata quale vera e propria riforma del processo matrimoniale, nonostante il Codice dica che una istruzione non è un atto legislativo ma un atto proprio della potestà esecutiva. Il fatto che essa sia stata emanata per espresso mandato del Supremo Legislatore e anche da Lui approvata in forma specifica, di per sé non dovrebbe cambiare la sua natura di atto della potestà esecutiva, testo di rango inferiore alle leggi alle quali non potrebbe certamente derogare né potrebbe cambiare la sostanza dei prescritti del codice. 
strettamente processual-penalistiche - che abbiamo denominato "integrative" del processo contenzioso ordinario - sono molto poche e di tenore piuttosto sommario: in soli 12 canoni è contenuta la disciplina dell'indagine previa e l'evoluzione dell'intero processo sino alla sua conclusione. La difficoltà è ancora maggiore se si considera che le norme speciali vanno composte e armonizzate con il processo ordinario ${ }^{11}$ : ciò richiede nel giudice canonico una perizia non comune nella conduzione di un processo penale. Il giudice ecclesiastico, che ha in genere maggiore confidenza con i processi matrimoniali più che non con quelli penali, di numero irrisorio rispetto a questi ultimi, certamente troverà poco agevole la celebrazione di un processo penale, se non è più che ferrato in materia. Sic stantibus rebus, appare chiaro quanto poco agevole sia per il giudice canonico la corretta celebrazione di un processo penale, senza un codice di rito che ne disciplini esattamente i singoli atti e le singole fasi. La normativizzazione sommaria del processo penale si risolve naturalmente in un rischio concreto e costante di decisioni arbitrarie e puramente discrezionali ${ }^{12}$, spesso a detrimento del diritto di difesa della persona imputata.

L'ordinamento penale canonico, poi, non conosce un altro principio cardine dell'ordinamento costituzionale italiano: mi riferisco al principio dell'obbligatorietà dell'azione penale ${ }^{13}$. L'assenza dell'obbligo significa che l'autorità ecclesiastica ha facoltà di decidere se e quando esercitare l'azione penale, con il risultato di una evidente disparità di trattamento tra fedeli. Il mancato esercizio dell'azione penale in determinati casi non è un vantaggio né per l'accusato né per la comunità ecclesiale: infatti, il mancato accertamento della responsabilità penale comporta che le accuse rimangano tali per un tempo indeterminato, senza mai diventare prove ma esplicando ugualmente un effetto sanzionatorio che un'accusa non dovrebbe e non potrebbe mai avere, se non verificata nel foro competente.

11 Can. 1728, § 1: "Salvis praescriptis canonum huius tituli, in iudicio poenali applicandi sunt, nisi rei natura obstet, canones de iudiciis in genere et de iudicio contentioso ordinario, servatis specialibus normis de causis quae ad bonum publicum spectant".

12 Per i problemi che pone l'ampia discrezionalità di cui gode il giudice canonico, si può utilmente consultare: P. AMENTA, La discrezionalità del giudice canonico e la tutela dei diritti del fedele cristiano, in D. CITO (a cura di), Processo penale e tutela dei diritti nell'ordinamento canonico, Giuffrè, Milano, 2005, 393-413.

13 Art. 112 Cost. Nella riforma del diritto penale canonico, che sta per entrare in vigore, si è fatto un piccolo passo avanti in questo senso, introducendo una norma che riguarda l'obbligo di comunicare le notizie di reato. 
Ciò si risolve nella negazione pratica del diritto della comunità ecclesiale ad accertare debitamente le situazioni di devianza e a porvi rimedio, nella negazione pratica del diritto del fedele a difendere la propria onorabilità nel foro competente della Chiesa e nella mortificazione che a lui proviene dallo stigma del sospetto di colpevolezza che si estende per un tempo indefinito, con i disagi facilmente immaginabili ${ }^{14}$.

Il rischio della disparità di trattamento non si ferma alla mancanza dell'obbligo di esercitare l'azione penale. Il rischio è accresciuto se si considera che manca nell'ordinamento canonico una chiara prescrizione analoga a quella dell'art. 25 della nostra carta costituzionale, il quale prevede la soggezione del cittadino accusato di reato al giudice naturale precostituito dalla legge. I giudici dello stato assumono il loro incarico dopo aver dimostrato con regolare concorso le proprie competenze e il loro incarico è stabile nel tempo: eventuali provvedimenti sanzionatori a loro carico sono emanati dal solo organo di autogoverno dei magistrati, il Consiglio Superiore della Magistratura. I giudici, dunque, sono assunti per competenza, sono stabili nell'incarico e indipendenti dalle autorità politiche o di governo. In ambito canonico, è possibile rinvenire una norma, quella del can. 1420, § 1 , che primo ictu oculi sembra soddisfare l'esigenza che ciascun imputato sia soggetto a un giudice precostituito dalla legge: si prescrive che ogni vescovo costituisca nella propria diocesi un vicario giudiziale con potestà ordinaria in ambito giudiziario. A lui, che esercita a nome del vescovo l'amministrazione della giustizia, possono essere assegnati giudici supplementari, oltre a un promotore di giustizia/difensore del vincolo (analogo al pubblico ministero italiano). Tuttavia, pur prescrivendo il codice determinate qualità essenziali per costituire i giudici ${ }^{15}$, nella pratica essi spesso non hanno titoli accademici specifici, sono liberamente nominati dal vescovo e non hanno un ufficio stabile perché nominati per un tempo determinato, come recita peraltro il

\footnotetext{
14 Analogamente avviene nell'ambito della potestà esecutiva: mi è noto il caso di una associazione di fedeli, ingiustamente accusata, deferita al Pontificio Consiglio per i Laici (ora denominato per la Famiglia e i Laici) il quale, a sua volta, ha deferito la questione alla Dottrina della Fede, che non ha mai risposto all'interpellanza del Pontificio Consiglio. Quest'ultimo, a cui sono giunte evidenze concernenti la falsità delle accuse, ritiene di non poter agire senza il parere della Congregazione. La situazione è di stallo. E intanto la vita di quei fedeli, e non sono pochi, è continuamente segnata da posizioni altalenanti dei vescovi nelle cui diocesi sono presenti, proprio in assenza di un provvedimento chiaro. E tutto ciò perché le eventuali risposte da parte degli organi di governo non sono disciplinati da alcuna legge, non esiste l'obbligo legale di rispondere e l'opportunità di una risposta è lasciata interamente alla discrezione dell'organo adito.

15 Cfr. can. $1420, \S 4$, e can. $1421, \S 3$.
} 
can. 1422. Dunque, i giudici ecclesiastici hanno un minore grado di stabilità e di indipendenza rispetto ai giudici dello stato; ma soprattutto non è sempre garantita dall'ordinamento la loro precostituzione: in ambito penale, ad esempio, che è peraltro quello più delicato, se un vescovo vuole procedere a celebrare un processo penale giudiziario e non ha un proprio tribunale diocesano, ipotesi prevista dal can. 1423, § 1, deve deferire il caso al tribunale di prima istanza costituito in solido con altri vescovi; ma è anche possibile che possa costituire un tribunale ad hoc, assegnando ad casum al proprio vicario giudiziale altri giudici che possano costituire un tribunale collegiale, secondo il prescritto del can $1425, \S 1$, sub $2^{\circ}$. Non è chi non veda che, in tali casi, la precostituzione per legge del giudice naturale è del tutto assente, con i rischi connessi alla costituzione del tribunale successiva alla commissione del reato. In ambito penale canonico, la competenza del tribunale è stabilita ratione loci in quo delictum patratum est (can. 1412): tuttavia la formulazione del canone è tale da far intravvedere come si possano ammettere altri titoli di competenza in materia penale, senza che questi siano definiti da alcuna norma espressa ${ }^{16}$. Ora, tal maniera di organizzazione giudiziaria va in senso contrario alla regola del giudice precostituito per legge, nata a presidio della indipendenza e della imparzialità del giudice: nel diritto canonico, mancando la regola espressa, di conseguenza non si può parlare di assoluta imparzialità del giudice.

La costituzione di tribunali speciali che nella Chiesa si occupino di casi determinati, se non è una regola costante, è certo un accadimento piuttosto frequente e ancor più evidente a livello universale. È a tutti noto che negli stati di diritto è sancito espresso divieto di costituire una magistratura speciale: anche questo divieto è, al pari di altri, stabilito a garanzia dell'indipendenza e dell'imparzialità della magistratura e a garanzia di una applicazione uniforme della legge. Infatti, la nostra costituzione proibisce la costituzione di tribunali speciali e con buon motivo: nella storia passata e recente, i tribunali speciali sono sempre stato sintomo di una giustizia d'urgenza, soggetta all'onda emotiva di questo o quell'evento, quando non soggetta a determinate scelte di politica criminale. Nella Chiesa, soprattutto a livello centrale, la garanzia di indipendenza e di equanimità è del tutto compromessa dalla costituzione di tribunali speciali. È a tutti noto, ad esempio, come papa Giovanni Paolo

16 Il can. 1412 recita: " [...] accusatus conveniri potest coram tribunal loci in quo delictum patratum est". Dunque, non si tratta di una regola tassativa e l'uso del verbo "potest" indica che vi possono essere altre possibilità: quali esattamente però il codice non lo rivela. 
II abbia costituito presso la Congregazione per la Dottrina della Fede un tribunale speciale per i delitti a sfondo sessuale (pedofilia, pedopornografia, abuso sessuale, assoluzione del complice in peccato turpi), dotandolo peraltro di una legge peculiare, nota come motu proprio De gravioribus delictis, del 2001, aggiornata poi da papa Benedetto XVI nel 2010. Ora, mettendo da parte la pur problematica commistione tra funzione amministrativa e funzione giudiziaria ${ }^{17} \mathrm{e}$ rimanendo nell'ambito strettamente processual-penalistico, se la costituzione di un tribunale speciale per determinati reati ha risolto da una parte l'esigenza di contare su personale qualificato, dall'altra il provvedimento suggerisce una scelta di "politica criminale", cioè di assicurare agli imputati di reati dall'ordinamento ritenuti più gravi (reati a sfondo sessuale soprattutto) un giudizio di particolare accuratezza e severità, con una diminuzione significativa delle garanzie per gli imputati ${ }^{18}$. Se a questo si aggiunge la

17 Le Congregazioni della Curia Romana sono di per sé emanazione del potere di governo, dunque organismi amministrativi (cfr. can. 360 e artt. 1 e 18, $2^{\circ}$ comma Cost. Apost. Pastor Bonus). Per giustificare l'emanazione di atti legislativi o di portata vincolante e l'esercizio di poteri giurisdizionali, recentemente persino la presenza, all'interno della Congregazione per la Dottrina della Fede, di un tribunale, i canonisti hanno affermato che la Congregazione di cui trattasi avrebbe conservato nel tempo le sue originarie competenze giudiziarie, risalenti al tempo in cui i Pontefici riservavano a sé la presidenza del Dicastero (cfr. V. DE PAOLIS, La Congregazione per la Dottrina della Fede, in Ephemerides Iuris Canonici, vol. 50 (2010). Ma se in presenza del Pontefice l'organo mutuava da Lui e dalla sua plenitudo potestatis anche le competenze di carattere giudiziario, oggi tale competenza non è più giustificabile e appare francamente un anacronismo, quantunque giustificato con apposite disposizioni legislative. Non manca neppure chi ammette una riserva di giudizio e di procedura alla Dottrina della Fede in materia di delicta (appunto) reservata e nel contempo ammetta la concorrenza con la giurisdizione dei tribunali locali, che non è tale perché i tribunali locali hanno solo una competenza funzionale in ordine a provvedere all'istruttoria delle cause deferite per competenza esclusiva alla S. Sede. Non si tratta dunque di un concorso di competenza veri nominis, che invece - erroneamente a mio giudizio - un autore sembra ammettere (cfr. D. SALVATORI, La riserva di alcuni delitti alla congregazione per la Dottrina della Fede e la nozione di delicta graviora, in Quaderni di diritto ecclesiale, 25 (2012), pp. 260-280, specialmente p. 277). Oggi, che il Pontefice non presiede nessuno dei Dicasteri della Curia Romana, di per sé ogni Dicastero potrebbe rivendicare a sé una competenza giudiziaria, mutuandola dalla pienezza della potestà del Romano Pontefice, nel nome del quale ogni Congregazione della Curia Romana opera.

18 Tra i provvedimenti del motu proprio Sacramentorum sanctitatis tutela che indeboliscono le garanzie della difesa ricordiamo l'eclatante provvedimento che prevede l'elevazione da 10 a 20 anni della prescrizione dell'azione penale in materia di delitti contra mores, da computare a partire dal compimento della maggiore età della vittima nei reati di abuso sessuale di minori. Nec sufficit! Una norma successiva (art. 7, § 1, del testo novellato nel 2010) prevede addirittura la facoltà che la Congregazione attribuisce a se 
mancata pubblicazione delle norme speciali emanate in materia e alla decisione di rimettere gli accertamenti non solo al foro giudiziario, ma anche al foro amministrativo, non c'è dubbio che tale legislazione sia stata emanata con l'intento di agevolare l'aspetto repressivo e punitivo, lasciando in ombra l'aspetto riparatorio ed educativo della legge ${ }^{19}$.

Un altro dei problemi che affliggono la celebrazione dei processi penali in ambito canonico, anzi, forse il problema principale, è l'assenza della possibilità di effettuare un esame incrociato. Di derivazione anglosassone, la cross examination costituisce un metodo di indagine ormai comunemente acquisito nella quasi totalità degli ordinamenti penali moderni: l'esame incrociato di parti, testi e periti da parte di accusa e difesa. L'esame incrociato, com'è noto, permette che la pubblica accusa e la difesa operino in assoluta parità potendo ciascuno interrogare la parte ed esibire prove documentali e d'altro genere a carico o a discolpa dell'accusato. Ora, se si consideri il fatto che le possibilità di indagine preliminare in ambito canonico sono molto limitate, la carenza di un esame incrociato impoverisce l'istruttoria dibattimentale e il potere di azione della difesa, il cui compito rimane marginale, limitato ai rilievi che possono essere compiuti in sede di difesa finale, a indagini ormai concluse. Il risultato è un apparato probatorio insufficiente a delineare le circostanze dei delitti, la credibilità dei testi e la responsabilità effettiva degli accusati. Questo accade anche perché il sistema canonico ammette solo teoricamente il principio dell'oralità: il processo penale canonico, come tutti gli altri, è un processo scritto. L'adozione della forma orale è ammessa come possibilità residuale e limitata ad alcuni atti singoli, estranea pertanto a intere fasi del processo $^{20}$. Ora, se si consideri che il principio di oralità è strettamente legato all'immediatezza con cui le prove sono acquisite in presenza dell'organo giudicante e della concentrazione

stessa di derogare alla prescrizione ventennale, con l'evidente risultato che un reato di tal genere risulti sempre e comunque perseguibile, anche a distanza di 40 anni! Per questi e molti altri rilievi critici, è utile consultare lo studio di D. MILANI, Delicta reservata seu delicta graviora: la disciplina dei crimini rimessi alla competenza della Congregazione per la Dottrina della Fede, in Stato, Chiese e pluralismo confessionale, Rivista telematica, n. 32 del 2013, pp. 1-25.

${ }_{19}$ Costituisce un'ulteriore conferma che tale sia la mens legislatoris il disposto del can. $1362, \S 1,2^{\circ}$, del codice penale novellato da papa Francesco, di imminente vigenza, che opera il passaggio della prescrizione ventennale dalla legge speciale prevista per la Congregazione al codice di diritto canonico e quindi alla legislazione generale.

20 Per tutta la materia lo studio fondamentale rimane ancora quello di A. NICORA, Il principio di oralità nel processo civile italiano e nel diritto processuale canonico, Pontificia Università Gregoriana, Roma, 1977. 
delle prove in un tempo limitato, cioè nella fase dibattimentale avanti al giudice che ha l'onere di emettere la sentenza, è chiaro che la scelta nettamente preferenziale che l'ordinamento canonico mostra per la forma scritta compromette sensibilmente da una parte l'immediata evidenza delle prove che il giudice ottiene dal dibattimento, dall'altra ingessa la parte imputata e la sua difesa, in quanto non è possibile proporre contestazioni a interrogazione diretta di parti e testi ${ }^{21}$. Il codice canonico prevede che solo il giudice interroghi, l'intervento del ministero pubblico non è previsto, quello della difesa è limitato: l'avvocato, se ha domande da proporre, deve porgerle al giudice il quale decide se la domanda è pertinente e decide se porgerla alla parte o al teste in esame ${ }^{22}$. Tale sistema, coerente peraltro con il modello inquisitorio, che ritengo essere ancora il modello di riferimento prevalente del processo penale canonico ${ }^{23}$, rallenta notevolmente il ritmo del processo, indebolisce il potere di autodifesa dell'imputato e allunga i tempi del processo, atteso il fatto che il giudice dovrà assumersi l'onere di leggere tutto il processo prima di emettere la sentenza.

\section{4 - Un esempio paradigmatico: il processo per l'imposizione di un decreto penale}

A conclusione delle nostre riflessioni, procediamo a una sorta di verifica sul campo di quanto finora abbiamo annotato. Anche il codice canonico conosce, al pari del codice processuale penale italiano, un procedimento

${ }^{21}$ Cfr. can. 1533 CIC83.

$22 \mathrm{Si}$ vedano i cann. 1530 e, specialmente, 1561 CIC83.

${ }^{23}$ È noto che i modelli processuali sono analoghi alle forme di governo che li generano e riflettono i valori che la comunità dei consociati condivide. Questo però non accade sempre. Talvolta si assiste a uno scostamento tra i valori condivisi e il sistema penale, e i fattori possono essere tanti e svariati, non ultimi le mancate riforme che avrebbero dovuto eliminare gli anacronismi del sistema. Per questo ho voluto dedicare un intero paragrafo di una mia ricerca a un tentativo di qualificazione del modello penale e processuale canonico. Tema scabroso che i canonisti - per quanto io ne sappia - hanno mai voluto sollevare, per i fantasmi che evoca il termine "inquisizione". Eppure, sono convinto, analizzando i caratteri del sistema processuale canonico, che esso sia un sistema molto vicino al modello inquisitorio, con l'introduzione di qualche marginale elemento accusatorio (P. AMENTA, Diritto processuale penale civile e canonico: due sistemi a confronto e tutela del diritto alla difesa, tesi di laurea in Giurisprudenza presso l'Università "Giustino Fortunato" di Benevento, anno accademico 2020-2021, non ancora pubblicata, specialmente capo III, § 3, pp. 61-69). 
per l'imposizione di un decreto penale. Di esso si parla ai cann. 1718, § 1 , sub $3^{\circ}$, e al can. 1720 . Nonostante il sistema canonico accordi una evidente preferenza per il processo giudiziario ${ }^{24}$, che assicura una maggiore profondità e serietà d'indagine e maggiori garanzie per la difesa dell'imputato, la mancanza di personale altamente qualificato e la necessità di chiudere quanto prima vicende scabrose e imbarazzanti, ha fatto sì che, soprattutto negli ultimi tempi e a seguito delle ben note vicende, nella Chiesa si adotti sempre più frequentemente il più snello $\mathrm{e}$ agile processo denominato per decretum extra iudicium, cioè il processo per $l^{\prime}$ 'imposizione di decreto penale ${ }^{25}$. In realtà, la tentazione d'intraprendere scorciatoie per arginare fenomeni emergenti, come può essere quello della pedofilia anche all'interno delle strutture ecclesiastiche, è sempre dietro l'angolo: ma è un sentiero non privo di rischi, soprattutto per il sacrosanto diritto all'autodifesa.

Il testo del can. 1342, $\S 1$, permette che si ricorra al decreto stragiudiziale per imporre una sanzione penale qualora ricorrano iustae causae. L'espressione è talmente generica che praticamente consente di contemplare qualsiasi motivazione plausibile e difficoltà di ogni genere e specie: la scarsa preparazione dei giudici e degli operatori del diritto in ambito processuale e penale, una certa tendenza alla deflazione del carico di lavoro dei tribunali, l'impossibilità di procurare mezzi di prova tipici

${ }^{24}$ Ciò è evidente non solo dal tenore di alcune norme del codice, ma anche dall'esame delle discussioni avvenute in sede di riforma dalla Commissione a suo tempo incaricata per la revisione del Codice: per tutto ciò si può utilmente consultare lo studio di G. PUNTILLO, Decreto penale extragiudiziale canonico e diritto di difesa: contributo alla comparazione con l'ordinamento italiano, Theses ad doctoratum in iure canonico assequendum, Lateran University Press, Città del Vaticano, 2010.

25 Si tratta di una tendenza di cultura giuridica non estranea neppure ai sistemi processual-penalistici statali: un filone importante della cultura giuridica del nostro tempo, che la dottrina ha denominato «tendenza alla destrutturazione» degli istituti giuridici, soprattutto quelli processuali. Un documento della Giunta dell'Unione delle Camere Penali italiane, datato 28 ottobre 2008, significativamente in proposito afferma: "Il codice di rito penale ha subito - e continua purtroppo a patire - nel tempo una graduale, inesorabile, destrutturazione che rischia sempre più di stravolgere l'impianto processuale di tipo accusatorio, a opera di sottosistemi che hanno una loro accentuata autonomia pur essendo inseriti all'interno del corpus codicistico" (cfr. Doppio binario:una deriva autoritaria del processo su cui iniziare una seria riflessione, reperibile in www.ristretti.it/commenti/2008/novembre/pdf1/ucpi_doppio_binario.pdf). Tale osservazione si raccorda con quanto abbiamo già espresso circa la mancata riforma globale del codice penale italiano. Si tratta, comunque, di una tendenza culturale, quella della destrutturazione degli istituti giuridici, che non risparmia le strutture della Chiesa cattolica e che rischia di generare ulteriori distorsioni patologiche del sistema. 
dei processi penali degli stati per l'assenza di un corpo di polizia giudiziaria, e via dicendo. Mancano, nel disposto di legge, altri importanti elementi di garanzia per l'imputato: non si prevede alcuna consultazione né l'imputato può operare una scelta del rito a cui assoggettare il proprio caso. La scelta è di esclusiva competenza del Superiore ecclesiastico. Dunque, un imputato non può chiedere, se non in via confidenziale, l'adozione del rito giudiziario, per una maggiore garanzia della propria difesa; né esiste alcuna facoltà per l'imputato di impugnare la decisione del Superiore, che sarebbe comunque improponibile, vista l'assenza di criteri tassativi o almeno indicativi per l'adozione di un rito piuttosto che di un altro. Soprattutto, ed è la differenza di maggior rilievo, rispetto al correlativo istituto penalistico del codice di procedura italiano, il procedimento per imposizione del decreto penale non è limitato ad alcune fattispecie delittuose: non esiste un elenco esemplificativo e ancor meno tassativo che prescriva quando e per quali reati sia possibile l'adozione del rito per l'imposizione di decreto penale. Di conseguenza, ogni fattispecie di reato, dalla più lieve alla più grave, può essere trattata con il procedimento per decreto, qualora il Superiore lo decida.

Procediamo ora per sommi capi a un'analisi comparatistica con il correlativo istituto processuale penale italiano per stabilirne $\mathrm{i}$ punti di contatto e le differenze, sempre operando una lettura che tenga conto delle garanzie individuali fondamentali delle persone coinvolte nel processo, in modo particolare la persona dell'imputato.

Quali sono i caratteri del processo per decreto nel sistema canonico? È possibile accostarlo alla tipologia presente nel codice di procedura penale italiano? E quali garanzie di ricerca dei mezzi di prova - e dunque di accertamento del fatto criminoso - possiedono tali istituti giuridici? Quale il grado di tutela dei diritti individuali, soprattutto il diritto di difesa?

Provo a dare una risposta sommaria alle domande appena esposte. Processo giudiziario e processo così detto amministrativo nel sistema canonico presentano differenze sia di ordine formale che di ordine sostanziale. Il processo giudiziario dovrebbe garantire un contraddittorio, almeno dal punto di vista formale, dato dalla presenza delle due figure di riferimento: il promotore di giustizia, titolare della pubblica accusa, e il patrono della parte imputata. Ambedue queste figure mancano del tutto nel processo canonico per decreto stragiudiziale. Esso non prevede alcuna forma di dibattimento. Il giudice è al tempo stesso il superiore dell'accusato, con un grave pregiudizio sulla terzietà e imparzialità del giudice. L'indagine previa non è affidata alla pubblica accusa ma a un auditor che ha il compito di raccogliere le prove indiziarie e trasmetterle al 
superiore. L'assenza del promotore di giustizia compromette la ricerca di idonei mezzi di prova: peraltro, il disposto del can. 1717, $\S 1$, sembra faccia riferimento non alla ricerca di indizi e mezzi di prova quanto piuttosto alla ricerca di indizi e notizie circa la verosimiglianza della notitia criminis. Sic stantibus rebus, appare sorprendente che il can. 1720, il disposto principale in materia, trattando della contestazione dell'accusa, parli espressamente di probationes. Come considerare prove l'accusa unita a indizi, atte a rendere verosimile l'accusa ma non certo sufficienti a emettere un decreto di condanna? La terminologia qui tradisce l'orizzonte mentale in cui si muove il legislatore canonico, ancora legato a una visione inquisitoria del processo: l'accusato è chiamato reus, già colpevole prima che gli vengano contestate le accuse e presentate le prove! Il decreto penale eventuale è dunque emanato sulla base di un procedimento sommario, operato principalmente sulla base degli atti raccolti nell'indagine preliminare, di cui ai cann. $1718-1719$. Il can. 1720 , al $\S 1$, sub $1^{\circ}$, tratta dell'obbligo fatto al superiore di offrire all'imputato la facoltà di difendersi. Un'espressione che risulta un'affermazione di principio, dal momento che, in assenza di una norma espressa che prescriva un interrogatorio formale dell'imputato $^{26}$, l'unica difesa possibile per l'accusato sarebbe quella che avviene alla presenza del superiore e, senza una preparazione dovuta alla mancata conoscenza degli atti, limitata alla facoltà di negare gli addebiti. Sembra dunque che la possibilità di difendersi da parte dell'accusato si riduca a una mera presenza dinanzi all'Ordinario o al suo delegato per ascoltare le accuse mossegli ed eventualmente contestare verbo, cioè seduta stante, gli elementi di accusa raccolti contro di lui, senza che all'accusato sia concesso alcun tempo per preparare una difesa e, sulla base della conoscenza precisa delle accuse, addurre documenti o altri elementi di controprova. Una comunicazione orale, senza poter operare un esame approfondito del materiale probatorio e senza alcun termine a difesa, come invece prevede per esempio il codice di procedura penale italiano ${ }^{27}$,

26 A onor del vero, il codice canonico non prevede neppure, in questo tipo di procedimento, una vera e propria contestatio litis, nella quale poter precisare la natura delle accuse e la loro ascrivibilità a un delitto formale e ammesso dal codice, con il rischio che il superiore ritenga delitto una contravvenzione o una semplice imprudenza la rivelazione del segreto confessionale, decidendo di deferire l'imputato alla Congregazione per la Dottrina della Fede, esclusivamente competente in materia. O ancora ritenere che una imprudenza o una proposta indecente avvenuta in un colloquio provato, per quanto moralmente censurabili, siano assimilabili a una sollicitatio ad turpia, che invece è un formale delitto, e tra i più gravi, ammessi dal codice di diritto canonico.

${ }^{27}$ Cfr. art. 519 c.p.p. 
non può chiamarsi possibilità effettiva di esercitare il proprio diritto all'autodifesa. Il silenzio del codice circa la presenza dell'avvocato in questa fase del processo e per tutta la sua durata, completa un quadro di evidente insufficienza del diritto alla difesa nel processo così detto amministrativo in ambito canonico. Manca peraltro nel codice canonico una procedura elaborata per l'esame dei ricorsi gerarchici ${ }^{28}$ : anche tale lacuna potrebbe avere effetti problematici sul diritto alla revisione delle decisioni non strettamente giudiziarie.

Per i motivi esposti e per tanti altri che brevitatis causa siamo costretti a omettere, non si può non concludere che il processo canonico per l'imposizione di un decreto penale non offre garanzie sufficienti quanto al diritto di difesa. La sua affermazione espressa si esaurisce in una affermazione di stile, senza effetto nella pratica. A riprova di quanto vado dicendo, segnalo che tale insufficienza non è certamente sfuggita al legislatore il quale ha trasfuso nel Codice dei canoni delle Chiese orientali, di qualche anno posteriore al Codice latino, una serie di norme correttive del tutto assenti in quest'ultimo. La legislazione per gli orientali testimonia dunque una sorta di "correzione di tiro", che non può non rivelare la chiara coscienza delle insufficienze del Codice latino in materia.

Il codice di procedura penale italiano contempla anch'esso un istituto che condivide con il codice canonico la denominazione procedimento per applicazione di un decreto penale. Ma la denominazione comune non deve trarre in inganno, perché, come presto si vedrà, i due istituti differiscono di molto, soprattutto per quanto concerne le garanzie per la parte imputata.

Il procedimento per l'applicazione di un decreto penale appartiene a quei riti speciali del codice di procedura che prevedono l'omissione del dibattimento, a cui appartengono anche altri riti, quello del giudizio abbreviato e quello dell'applicazione della pena su richiesta, il c. d. patteggiamento. Altri riti speciali invece prevedono l'omissione dell'udienza preliminare per passare immediatamente al dibattimento. Il giudizio immediato e il giudizio direttissimo. Dunque, l'applicazione della pena con decreto di condanna si caratterizza come procedimento senza dibattimento: il giudice emette il decreto sulla base degli atti che ha a disposizione, risultato delle indagini svolte dal pubblico ministero con l'ausilio della polizia giudiziaria. Sotto questo profilo, si può ritenere che questo sia un punto in comune con l'analogo procedimento canonico,

28 L'affermazione è di F. DANEELS, L'imposizione amministrativa delle pene ed il controllo giudiziario sulla loro legittimità, in D. Cito (a cura di), Processo penale, cit., p. 295. 
anch'esso caratterizzato dall'assenza di una fase di confronto tra accusa e difesa. A uno sguardo più attento, però, non sono poche né di poco conto le differenze tra l'istituto quale previsto dal codice di procedura italiano e quello presente nel codice di diritto canonico, appena delineato per sommi capi.

Anzitutto, il decreto penale nel sistema italiano è emesso al termine di un processo - sommario, ma pur sempre processo - che avviene nella sede naturale, quella giurisdizionale, davanti a un giudice, senza alcuna commistione con l'ambito amministrativo. Dunque è garantita, almeno in linea di principio, l'imparzialità del giudice precostituito per legge e assolutamente indipendente. In secondo luogo, il processo presenta aspetti di premialità, con scopi chiaramente deflattivi ${ }^{29}$, in quanto scoraggiano le opposizioni eventuali: aspetti del tutto assenti nel codice canonico. In terzo luogo, il processo per decreto può essere attivato solo su richiesta della pubblica accusa e senza l'opposizione del querelante, non può essere adibito per ogni specie di reato ma solo per i reati perseguibili d'ufficio o a querela di parte che prevedano l'applicazione di una pena pecuniaria in sostituzione di una pena detentiva. Quindi, in ultima analisi, solo per reati di minore entità. Inoltre, il decreto penale non è ammesso quando risulti necessaria l'applicazione di una misura di sicurezza personale ${ }^{30}$. L'assenza di una sola di queste condizioni rende inammissibile il procedimento. L'articolo di legge stabilisce accuratamente il modo con cui il giudice deve operare il calcolo della pena pecuniaria sostitutiva della pena detentiva, tenuto conto della condizione economica complessiva dell'imputato e il suo nucleo familiare. Non è chi non veda quanto i due istituti, quello penale civile e quello canonico, siano effettivamente distanti: il procedimento canonico non ha limiti e dunque almeno in teoria, può essere adibito senza alcuna cautela o scelta da parte di imputato o accusa per ogni tipo di reato, eccettuati solo quei pochissimi per i quali il codice canonico prescrive obbligatoriamente l'adozione del processo giudiziario. Non presenta aspetti premiali e non è prescritta alcuna tipologia di pena: teoricamente ogni tipo di pena è applicabile, a eccezione delle pene perpetue $^{31}$. Non è prevista alcuna sostituzione di pena con multa, né vi

29 L'art. 459, secondo comma, del c.p.p. stabilisce che il pubblico ministero "può chiedere l'applicazione di una pena diminuita sino alla metà rispetto al minimo edittale". L'art. 460, al quinto comma, stabilisce che il decreto penale di condanna non comporta la condanna al pagamento delle spese processuali né l'applicazione di pene accessorie.

${ }^{30}$ Cfr. art. 459 c.p.p., specialmente quinto comma.

${ }^{31}$ Cfr. can. $1342, \S 2$. 
sono criteri per decidere l'ammontare dell'eventuale risarcimento del danno che consegue all'offesa ${ }^{32}$.

Nonostante l'assenza del dibattimento e il giudizio operato sulla base dei soli atti raccolti dal pubblico ministero, nel procedimento penale statale il diritto di difesa è assicurato, oltre che dalla presenza di un difensore di fiducia o d'ufficio, anzitutto dalla facoltà di proporre opposizione contro il decreto, con contestuale richiesta di cambio del rito: l'imputato può accedere così al rito ordinario oppure a uno dei riti abbreviati. Inoltre, contro l'eventuale ordinanza di inammissibilità dell'opposizione da parte del giudice che emise il decreto è sempre possibile proporre un ricorso per cassazione ${ }^{33}$. Infine, è la legge stessa che prevede alla revoca del decreto penale di condanna, in presenza di una legittima opposizione.

Come si può osservare da quella sommaria elencazione delle caratteristiche dell'istituto processuale di imposizione di un decreto penale di condanna, di ben altro tenore sono le norme dello stato rispetto a quello canonico. Il processo, pur omettendo, almeno in una prima fase, il dibattimento, è congegnato in modo tale da non compromettere sostanzialmente il diritto alla difesa costituzionalmente garantito: tale è stata l'affermazione della Corte costituzionale, a seguito delle numerose questioni di costituzionalità del procedimento sollevate. Sono numerose le sentenze nelle quali la Consulta spiega i motivi per cui il processo per decreto non compromette l'integrità degli artt. 3, 243111 della Carta costituzionale. Seppure non possa parlarsi, nel caso, di un integrale e perfetto diritto di difesa, assicurato solo dal processo ordinario, è indubbio che il processo penale per decreto previsto dal codice italiano di procedura rispetti alcuni elementi di non poco conto, riconducibili a un sostanziale esercizio del diritto alla difesa: la facoltà di scelta o di mutamento del rito, la facoltà di chiedere il contraddittorio, di fare opposizione al decreto o ricorso contro lo stesso, di accedere agli atti di causa per un esame approfondito con l'ausilio di un legale. Garanzie che non è possibile rinvenire nel Codice canonico. Resta solo la speranza - nell'attesa di poter adeguare le affermazioni di principio con un codice procedurale che preveda meccanismi concreti di tutela del diritto alla propria difesa - che le lacune della legge siano colmate dalla giurisprudenza. Ma una

\footnotetext{
32 L'assenza di tali garanzie di legge e l'amplissima discrezionalità lasciata al giudice o al superiore ecclesiastico nella determinazione della pena e di eventuali accessori può dar luogo a vere e proprie aberrazioni, che ripugnano al più elementare senso di giustizia.

${ }^{33}$ Cfr. art. 461, sesto comma, c.p.p.
} 
giurisprudenza corretta e rispettosa dei principi fondamentali della persona umana può essere formata solo da personale preparato, che sappia contemperare la salvaguardia degli interessi della comunità con gli interessi e i diritti della persona umana, memori dell'adagio terenziano sempre valido: homine imperito numquam quidquam iniustius qui, nisi quod ipse fecit, nihil rectum putat.

La proclamata "elasticità" del diritto ecclesiale, pienamente giustificata per una società multiculturale così vasta per alcune materie, non doveva coinvolgere anche l'aspetto processuale penale, che presiede alla tutela dei diritti fondamentali della persona umana, validi dunque per il fedele di qualunque cultura e latitudine; anzi, al contrario, come afferma un insigne canonista:

"in ambito canonistico, l" elasticità' e l'affievolimento del principio di legalità penale, previsti dal can. 1399, devono essere compensati con una particolare cura della legalità processuale $[\ldots]$ nel tentativo di rendere operativa la tutela dei diritti dei fedeli sanciti dai Principi per la riforma del CIC approvati dal Sinodo del 1967, come fu riconosciuto dallo schema del 1973 sul diritto penale" ${ }^{\prime \prime 34}$.

Fino a ora, l'auspicio del Prof. Llobell non può certo dirsi compiuto.

${ }^{34}$ J. LLOBELL, Contemperamento tra gli interessi lesi ed i diritti dell'imputato. Il diritto all'equo processo, in D. CiTO (a cura di), Processo penale, cit., p. 72. 